\section{Has covid-19 precipitated the end of the family doctor?}

\author{
Kieron Cooney senior GP Partner
}

The covid pandemic has precipitated many changes to how general practitioners have had to work, raising debate about the future of traditional general practice and concerns about the likely demise of the "family doctor." I have been thinking about the question of how much value there is in the family doctor model of primary care and whether patients, or indeed newly qualified GPs, appreciate what it is we risk losing if we move away from this model.

I have been a GP in one practice for 30 years and it has been my privilege to grow and develop alongside my patients as a family doctor.

I believe it is a model of primary care practice that allows us to provide holistic and family medicine to our patients with a pastoral sense of connection with families. We have enjoyed trust, respect, and friendship with a sense of community belonging. It may not quite be Dr Finlay's casebook, but like the TV series about Finlay's general practice in the fictional town of Tannochbrae, I have also seen families throughout their lives, providing medical care and support through many milestones such as births, deaths, marriages, divorces, and much more along the way. I believe I have a sense of connection with the community through family contacts and sustained professional relationships, providing continuity of care to my patients.

Needless to say, this approach to medicine has been a rewarding and fulfilling career path, one that I fear may now be in decline.

As a GP trainer and college examiner, I continue to support the Royal College of General Practitioners (RCGP) in their training for new GPs. This maintains an emphasis on the importance of supporting patients based on a holistic psycho social medical model.

I fear an ever growing move towards a short term problem solving model incorporating non face to face acute assessment, triage, and signposting to auxiliary primary care practitioners, such as advanced nurse practitioners, community practitioners, pharmacists, mental health workers, and care navigators. Home visits have all but disappeared through 111 support, leaving more vulnerable patients without easy access to their GPs. My patients are now faced with a patient handling service to redirect them to an appropriate service. This results in ever increasing difficulty in accessing their own family GP and leads many to express considerable frustration and disappointment, despite receiving a good clinical assessment from another practitioner. This strategy reflects the ever increasing demands of society on our primary care health service, outstripping the capacity for the family $\mathrm{GP}$, to provide family medicine support.

Despite my concerns, I do truly value all of these services for sharing and supporting the management of patients, without which, I would not be able to cope, especially with current pressures and workload demands. But what is the future impact on our professional relationship with patients of diluting our connection with them in this way?

Furthermore, our clinical encounters are defined by protocols and guidelines with obvious safety and consistency in management decisions in mind, though risking a formulaic and depersonalized approach to patients. Many practices are now contemplating a total triage system of practice, or an increasing use of electronic communication systems, further widening the gap between the GP and his patient.

These new systems have been driven by the increased demand and expectations on the service. The expectations from young families and individuals now seem to be for rapid access to advice and treatment with any available medical practitioner. Many practices now have no allocated personal list commitment so that consultations are with whomever is on the appointment list based on urgency, availability, or on a pre-planned arrangement with whichever doctor has the shortest waiting time. While it is understandable that patients' preferences have changed, this shift creates the potential for poor continuity of care, reactive medicine, and limited capacity for preventative or holistic care medicine.

These changes are also having an impact on how trainees perceive general practice. My own experience at recruiting partners, salaried or not, over recent times suggests that many newly qualified GPs no longer see general practice as a long term family doctor position, and have little interest in being committed to a partnership model. This is leading to a tangible loss of GP manpower and inevitable recruitment problems which may compromise the future of family practice medicine.

My own role as a GP in primary care remains exciting and rewarding with a commitment to long term patient continuity of care and support, alongside a determined interest in overseeing and being part of national primary care health improvement strategies, particularly where they are in keeping with the priorities of my own community needs for the benefit the local population.

As the number of family doctors decline I can only hope that a new generation of primary care practitioners will become the key drivers for future changes in the way the NHS is provided in primary care. The role of the GP of the future appears to be heading towards becoming a consultant for primary care, working alongside, and as part of, an ever increasing multidisciplinary team of primary care practitioners.

Ultimately I trust in the NHS to continue to provide excellent support and care at primary care level, but 
I cannot help having a strong sense of loss for the family doctor model that I have loved, and now miss as I approach the end of a long career. Whether the overall future health of our communities without the family doctor will be compromised or patients will feel this loss only time will tell.

Competing interests: none declared

Provenance and peer review: not commissioned, not peer reviewed 